Federal Reserve Bank of Minneapolis

Research Department

\title{
Is There a Stable Relationship between Unemployment and Future Inflation? Evidence from U.S. Cities*
}

\author{
Terry J. Fitzgerald and Juan Pablo Nicolini \\ Working Paper 713 \\ May 2014 \\ Preliminary and Incomplete
}

\begin{abstract}
This paper makes two straightforward points that we argue are central to understanding the literature and debate surrounding the stability of the Phillips curve. First, the endogeneity of monetary policy implies that aggregate data are largely uninformative as to the existence of a stable relationship between unemployment and future inflation. Second, if the NAIRU model is assumed to be true, regional data can be used to identify the structural relationship between unemployment and future inflation. We find that a 1 percentage point increase in the unemployment rate is associated with a roughly 0.3 percentage point decline in inflation over the next year.
\end{abstract}

Keywords: Endogenous monetary policy; Stability of the Phillips curve JEL classification: E52, E58

*Fitzgerald: Federal Reserve Bank of Minneapolis, tjf@minneapolisfed.org. Nicolini: Federal Reserve Bank of Minneapolis and Universidad Di Tella, juanpa@minneapolisfed.org. The research in this paper originated in a question posed to us by Narayana Kocherlakota. We especially thank Marco Bassetto for a very useful discussion. We also thank V.V. Chari, Constantino Hevia, Patrick Kehoe, Ellen McGrattan, Martin Sola, Pedro Teles, and Mike Woodford for helpful discussions and Brian Holtemeyer and Claire Hou for outstanding research assistance. The views expressed herein are those of the authors and not necessarily those of the Federal Reserve Bank of Minneapolis or the Federal Reserve System 


\section{Introduction}

This paper makes two straightforward points that we argue are central to understanding the literature that studies the stability of the Phillips curve. First, the endogeneity of monetary policy implies that aggregate data used in the analysis throughout this literature are largely uninformative as to the existence of a stable relationship between unemployment and future inflation. Second, if the NAIRU (non-accelerating inflation rate of unemployment) model is assumed to be true, regional data can be used to identify the structural relationship between unemployment and future inflation. The empirical analysis of the regional data is remarkably consistent with the stability of the parameters in the assumed model. In addition, assuming the NAIRU model is true, we find that a 1 percentage point increase in the unemployment rate is associated with a roughly 0.3 percentage point decline in inflation over the next year. Interestingly, this estimate is quite similar to estimates claimed by some NAIRU proponents several years ago. ${ }^{1}$

Using a standard NAIRU-type model in which the central bank's objective is to stabilize inflation around a target rate, we first show that aggregate data are uninformative regarding the true structural relationship between unemployment and future inflation. We show that under a specific and reasonable definition of inflation targeting, the evolution of equilibrium inflation is a random walk. ${ }^{2}$ Thus, no forecasting rule that uses a measure of unemployment, or any other variables for that matter, outperforms the naive rule that inflation next year will be the same as inflation last year. ${ }^{3}$ The reasoning behind this result is straightforward. Given the assumptions regarding central bank behavior, policymakers will do whatever they can to keep the equilibrium inflation rate from departing from its specified target. Therefore, the

\footnotetext{
${ }^{1}$ See, for instance, Reifschneider, Tetlow, and Williams (1999) who use a value of 0.4.

${ }^{2}$ This result is the same as the one in Nicolini (2013), but in a very different context.

${ }^{3}$ This is the rule used by Atkeson and Ohanian (2001) in their influential paper.
} 
difference between inflation and the target will be the result of unforecastable from the central bank's viewpoint-shocks. Equilibrium inflation will be equal to the exogenous target plus shocks that are uncorrelated with variables in the information set of the central bank.

More generally, we show that if the central bank has a dual mandate on inflation and employment, the statistical relationship between unemployment and future inflation growth depends on policy parameters such as the relative weights and values for the targets. We show that under changing policy regimes, our theory implies that the relationship between unemployment and inflation also changes. Atkeson and Ohanian (2001) and many others have provided a dramatic empirical demonstration of this point using data for the United States over the past 50 years.

Second, we show that if the NAIRU model is assumed to be true, the structural relationship can be identified by using regional data in a way that the theory makes very precise. The reason is that monetary policy stabilizes average inflation, so regional variation (which averages out nationally) can still be used to recover the structural parameter. We show that it is the difference between regional and average variables that should maintain a stable relationship in spite of changes in the policy regime. We present some simple regressions - suggested by our theory - that show that the NAIRU model clearly passes this stability test. Moreover, the number we obtain for the partial effect on future inflation generated by a 1 percentage point increase in unemployment is 0.3 percentage points, very close to the 0.4 figure claimed by NAIRU Old Keynesians.

A caveat is in order. Our analysis assumes that the NAIRU model is true and derives a simple empirical test that is not affected by policy shifts. The model passes the test in a remarkable way. Although this finding is consistent with the NAIRU model, it may also be consistent with other interpretations, as we discuss in more detail in the conclusions. So our results do not prove Phillips curve skeptics wrong. 
We simply argue that aggregate data are uninformative because policy is endogenous, and we show that regional data - which is immune to policy changes - show a robust and surprisingly stable relationship during the 1977-2010 period. This paper shows that aggregate data regressions should not be used to build skepticism or support for NAIRU models; in addition, regional data on their own do not provide clear evidence of parameter shifts.

\subsection{Background on the Phillips Curve}

The prominent role of central banks is to pursue price stability. On this account, the performance of the Federal Reserve since the disinflation of the early 1080s has been remarkably successful. During the last 30 years, inflation in the United States as measured by the personal consumption expenditures price index has averaged 2.4 percent a year, with a maximum 12-month rate of 5.1 percent in 1990 and a minimum rate of -1.2 percent in 2009. Furthermore, much of this volatility is associated with energy price movements. Over the last 20 years, core inflation measured on a 12month basis has remained in a remarkably narrow range between 1.0 percent and 2.5 percent.

In many countries, however, central banks are expected to do more than stabilize prices. In the United States, for instance, Congress has mandated that the Federal Reserve promote maximum employment as well as price stability. Countries in the euro area built their new central bank on a similar two-pillar strategy. Many other countries place employment or economic activity goals alongside the central banks' price stability role.

Although the mechanism by which a central bank can control long-run inflation is relatively well understood, the way in which monetary policy affects employment fluctuations is more controversial. Analysis of the relationship between inflation and unemployment dates back to Irving Fisher, who in 1926 documented a notable statis- 
tical relationship using data from the United States. The topic attracted much more attention when William Phillips published research in 1958 that showed a statistically significant negative relationship between unemployment and the growth rate of nominal wages: the Phillips curve. This statistical relationship was the foundation of the analysis in the work of Samuelson and Solow (1960), who argued that a looser monetary policy that allows rising inflation could reduce the unemployment rate for several quarters.

However, the notion that the statistical relationship implied a trade-off between inflation and employment that could be exploited by monetary policy was forcefully contested by the pathbreaking work of Lucas (1972), who provided a theoretical model without an exploitable trade-off but which was consistent with the existence of the observed statistical relationship. In Lucas, this statistical relationship depends on the parameters governing monetary policy, and in that sense it is not "structural." The Lucas model predicted that if monetary policy attempted to exploit the observed relationship, the Phillips curve would shift (i.e., the parameters would change), resulting in higher inflation without any effect on unemployment. The dramatic shift in the Phillips curve and the stagflation of the late seventies that followed Lucas's work gave strength to the notion that there is no exploitable trade-off between inflation and measures of real activity.

Although the Phillips curve lost favor in most academic circles, it never abandoned the corridors of many central banks, though it remained with some important caveats. The new version of the trade-off included the notion (known as the NAIRU hypothesis) that the real effects of a monetary policy are a short-run phenomenon. Models that include a NAIRU-type Phillips curve insist on the notion that monetary policy has predictable and structural effects on real activity.

The hypothesis of an exploitable Phillips curve, however, continues to be controversial. The influential paper by Atkeson and Ohanian (2001) shows that the empirical 
relationship between current unemployment and future inflation growth - assumed to be structural in NAIRU-type models - is highly unstable over the period 1960-2000 in the United States. They forcefully argue this point by showing that a naive prediction rule for inflation that simply uses past inflation is systematically better at forecasting than empirical NAIRU-type Phillips curves. This lack of forecasting ability is precisely the result of shifting parameters in the estimated Phillips curves, as the authors argue. Out-of-sample forecasts perform very poorly. Atkeson and Ohanian argue that the period covered by the analysis includes changing policy regimes, and the corresponding shift in parameters is evidence that the relationship is not structural. It follows, one could then argue, that policy cannot be used reliably to exploit a trade-off between inflation and unemployment even in the short run. The analysis of this paper provides a different interpretation of the data.

The paper is organized as follows. Section 2 develops the theory and shows the effects on the equilibrium outcome of assuming that the central bank operates as an inflation targeter in ways we make very precise. We develop the implications of the theory for the regional regressions and show the results using data from 27 metropolitan statistical areas (MSAs) in the United States from 1976 to 2010. As we show, the regressions are remarkably consistent with the notion that an increase in the current unemployment rate of 1 percentage point is associated with a decrease in inflation growth of roughly 0.3 percentage points. But this single-mandate version of the model is not consistent with a negative slope for the Phillips curve as identified using aggregate data over some time periods, as documented in the literature. Thus, in Section 3 , we generalize the model to incorporate a dual mandate and derive the theoretical implications. In this case, the estimated parameters for the aggregate regressions need not be zero, but depend on policy variables. It is still the case, however, that regional data can be used to identify the underlying parameter even in the presence of policy shifts, but the regressions for the MSAs are different. But this generalization does 
not change the stability of the relationship estimated between current unemployment and future inflation growth: the parameter is still consistently estimated to be around -0.3. The results of the regressions are also consistent with the assumption of policy shifts over time, as has been argued before (Atkeson and Ohanian (2001), Sargent (1999)). A final section provides some caveats and concluding comments.

\section{The Model with a Single Mandate}

Consider a single-good labor-only economy with a continuum of separated identical markets and price frictions in each market. All markets use the same unit of account and face the same monetary policy. Moving across locations is costly, and the shocks are small enough so that there is no migration in equilibrium. These assumptions imply that we can treat each region as a closed economy that shares a common monetary policy with all other regions. ${ }^{4}$

A very important caveat is in order. Modern macroeconomics is built on microfoundations. Old Keynesian (OK) models, such as the one we will describe, are not. ${ }^{5}$ The purpose of this paper is to discuss evidence on whether the NAIRU Phillips curve has shifted over time in the last half century or so. For this purpose, we fully embrace an OK NAIRU-type model without microfoundations and use it to derive simple empirical tests.

Simple OK NAIRU-type models are described by a Phillips curve and an investment saving (IS) curve. These two differential equations with the two endogenous variables (inflation and unemployment deviations) are then solved to obtain a solution for each

\footnotetext{
${ }^{4}$ These assumptions make the algebra very simple, but are by no means innocuous. See the discussion in Section 4.

${ }^{5}$ Cochrane (2011) makes an interesting comparison between what he calls Old Keynesian (OK) and New Keynesian (NK) models. We follow his terminology. A further comparison is made in Ayres and Nicolini (forthcoming).
} 
of the endogenous variables as a linear function of their lagged values.

Typically, the Phillips curve and the IS curve equations are called "structural equations," whereas the dynamic equations that describe the solution are called "reduced form" equations. In what follows, we will be interested in the parameters governing the reduced form equations for three reasons. First, from a policy point of view, it is the reduced form equation that determines how much inflation increases in the future if unemployment is reduced today because of, say, a monetary policy shock. Second, the reduced form equations are the ones that determine the moments in the data we discuss in this paper. Third, given the lack of microfoundations in OK models, we do not find the distinction between "structural" and "reduced form" particularly interesting. In Appendix B we describe the structural equations of a particular OK model - a very minor extension of the one presented in Taylor (1999) and discussed in Cochrane (2011) - that delivers the reduced form expressions that follow.

Let $\pi_{t}(j), u_{t}(j)$ represent regional inflation and unemployment. The NAIRU model solution in region $j$ is given by

$$
\begin{aligned}
& \pi_{t+1}(j)=A+B \pi_{t}(j)+C u_{t}(j)+F i_{t}+\varepsilon_{t+1}^{\pi}(j)+\xi_{t+1}^{\pi} \\
& u_{t+1}(j)=A^{\prime}+B^{\prime} \pi_{t}(j)+C^{\prime} u_{t}(j)+F^{\prime} i_{t}+\varepsilon_{t+1}^{u}(j)+\xi_{t+1}^{u},
\end{aligned}
$$

where $\varepsilon_{t}^{i}(j), \xi_{t}^{i}, i=u, \pi$ are the regional and aggregate shocks. We assume shocks have zero unconditional means, and the regional shocks are independent of the aggregate shock. The terms $F i_{t}$ and $F^{\prime} i_{t}$ describe the immediate effect of monetary policy on the two endogenous variables. The timing indicates that the monetary authority decides on policy before observing the $t+1$ shocks.

The key assumption in NAIRU models is that the parameter $C$ is negative. If prices were fully flexible, $C$ and $B^{\prime}$ are presumably zero, though the lack of microfoundations does not allow precise statements in this regard. ${ }^{6}$

\footnotetext{
${ }^{6}$ In the OK model described in Appendix B, the coefficient in the reduced form inherits the
} 
If we define aggregates as

$$
\begin{aligned}
\pi_{t+1} & =\int_{0}^{1} \pi_{t+1}(j) d j \\
u_{t+1} & =\int_{0}^{1} u_{t+1}(j) d j,
\end{aligned}
$$

we obtain the following relationship between the aggregate variables:

$$
\begin{aligned}
& \pi_{t+1}=A+B \pi_{t}+C u_{t}+F i_{t}+\xi_{t+1}^{\pi} \\
& u_{t+1}=A^{\prime}+B^{\prime} \pi_{t}+C^{\prime} u_{t}+F^{\prime} i_{t}+\xi_{t+1}^{u} .
\end{aligned}
$$

Our assumption of identical regions implies that we do not need to weight the regional values in order to obtain the aggregate, which considerably simplifies the algebra that follows. In particular, the parameter $C$ is the same for every region, so the solution for aggregate inflation in (1) also implies that higher current unemployment is associated with lower future inflation in each region.

The stability over time of that parameter $C$, particularly across different monetary policy regimes, has been the focus of much discussion in the literature. The ability to identify $C$ in the data is the focus of this paper.

\subsection{Exogenous Policy}

Assume that the monetary authority follows an exogenous policy. For the sake of argument, let $i_{t}=i$ for all $t$, for some $i>0$. Then, equilibrium inflation ${ }^{7}$ evolves as

$$
\pi_{t+1}=A+B \pi_{t}+C u_{t}+F i+\xi_{t+1}^{\pi}
$$

or

$$
\pi_{t+1}-\pi_{t}=B\left(\pi_{t}-\pi_{t-1}\right)+C\left(u_{t}-u_{t-1}\right)+\left(\xi_{t+1}^{\pi}-\xi_{t}^{\pi}\right) .
$$

(negative) sign of the coefficient in the structural form. In NK models, the structural coefficient is positive, rather than negative.

${ }^{7}$ We ignore some issues regarding price level determinacy in this subsection; see footnote 9 for more details. 
Clearly, under this policy, the equilibrium of the model exhibits a linear relationship between current unemployment and inflation growth. Thus, standard econometric techniques should suffice to identify the parameter $C$. In addition, current unemployment should help to forecast inflation changes. The evidence provided by Atkeson and Ohanian (2001) is inconsistent with a stable value for $C$ in this model.

\subsection{Endogenous Policy}

To start with the simplest case, imagine the central bank has a single mandate to stabilize inflation. In particular, imagine that the bank solves the following policy problem $^{8}$

$$
\min _{i_{t}} \frac{1}{2} E_{t}\left[\pi_{t+1}-\pi_{t+1}^{*}\right]^{2}
$$

given $\pi_{t}, u_{t}$, and the solution for aggregate inflation (1). The target for inflation is given by $\pi_{t+1}^{*}$. The optimal policy rule is ${ }^{9}$

$$
i_{t}^{O p t}=\frac{1}{F}\left[\pi_{t+1}^{*}-\left(A+B \pi_{t}+C u_{t}+E_{t} \xi_{t+1}^{\pi}\right)\right],
$$

so the equilibrium value for inflation is given by

$$
\pi_{t+1}=\pi_{t+1}^{*}+\xi_{t+1}^{\pi}-E_{t} \xi_{t+1}^{\pi}
$$

The behavior of equilibrium inflation depends on the behavior of the target for inflation, $\pi_{t+1}^{*}$, which is not observable. To gain further insight, we next consider several specifications for the inflation target. We then use these specifications in our empirical analysis.

\footnotetext{
${ }^{8}$ For a similar analysis in a different application, see Nicolini (2013).

${ }^{9} \mathrm{An}$ issue regarding uniqueness is related to this policy rule. We show in Appendix B that in the context of that particular OK model, this is not a problem. See also Cochrane (2011) for a discussion of determinacy in models of this type.
} 


\subsubsection{Constant inflation target}

If $\pi_{t}^{*}=\pi^{*}$, then

$$
\pi_{t+1}-\pi_{t}=\left(\xi_{t+1}^{\pi}-E_{t} \xi_{t+1}^{\pi}\right)-G\left(\xi_{t}^{\pi}-E_{t-1} \xi_{t}^{\pi}\right)
$$

so current unemployment would be related to the change in inflation to the extent that the forecast error $\left(\xi_{t}^{\pi}-E_{t-1} \xi_{t}^{\pi}\right)$ affects unemployment $u_{t}$. But if an estimate of the change in inflation different from zero is obtained, it is unrelated to the direct effect of unemployment on future inflation, or $C$.

\subsubsection{Inflation target with bands}

Next assume that the target for inflation is given by

$$
\begin{aligned}
& \pi_{t}^{*}=\pi_{t-1}, \text { if } \pi_{t-1} \in\left[\pi_{\min }, \pi^{\max }\right] \\
& \pi_{t}^{*}=\pi^{\max }, \text { if } \pi_{t-1}>\pi^{\max } \\
& \pi_{t}^{*}=\pi_{\min }, \text { if } \pi_{t-1}<\pi_{\min } .
\end{aligned}
$$

Thus, as long as the target remains within the band, $\pi_{t+1}^{*}=\pi_{t}$, then

$$
\pi_{t+1}-\pi_{t}=\xi_{t+1}^{\pi}-E_{t} \xi_{t+1}^{\pi}
$$

so inflation would be a random walk. In this case, current unemployment - or, for that matter, any variable in the information set at time $t$ - should not help predict inflation. Thus, according to this theory, the analysis in Atkeson and Ohanian (2001) is uninformative regarding the structural parameter $C{ }^{10}$

\footnotetext{
${ }^{10}$ This case is inconsistent with regressions using aggregate data delivering nonzero coefficients for some periods of recent U.S. history. On the other hand, it is hard to argue that this regime is a reasonable characterization of U.S. monetary policy for some of those periods, such as the seventies. We discuss this further below.
} 


\subsection{Regional Regressions}

We now show how regional data can provide useful information on the parameter $C$. The regional equations exploit the fact that the deviations of the regional variables with respect to the national average will not be correlated with policy, since policy responds, by assumption, only to the national average. Indeed, applying the optimal policy to the solution for each region, we obtain

$$
\pi_{t+1}(j)=\pi_{t+1}^{*}+B\left(\pi_{t}(j)-\pi_{t}\right)+C\left(u_{t}(j)-u_{t}\right)+\varepsilon_{t+1}(j)+\xi_{t+1}^{\pi}-E_{t} \xi_{t+1}^{\pi} .
$$

If the inflation target is constant, then the following regression,

$$
\pi_{t+1}(j)=\pi^{*}+B\left(\pi_{t}(j)-\pi_{t}\right)+C\left(u_{t}(j)-u_{t}\right)+\varepsilon_{t+1}(j)+\xi_{t+1}^{\pi}-E_{t} \xi_{t+1}^{\pi}
$$

allows us to identify the effect of unemployment in future inflation, $C$.

On the other hand, for the case of the inflation target with a band, and assuming inflation was always within the band, we obtain

$$
\pi_{t+1}(j)-\pi_{t}=B\left(\pi_{t}(j)-\pi_{t}\right)+C\left(u_{t}(j)-u_{t}\right)+\varepsilon_{t+1}(j)+\xi_{t+1}^{\pi}-E_{t} \xi_{t+1}^{\pi} .
$$

A final, more general specification would simply add a time effect to the regression

$$
\pi_{t+1}(j)=D_{t}+B\left(\pi_{t}(j)-\pi_{t}\right)+C\left(u_{t}(j)-u_{t}\right)+G \varepsilon_{t+1}(j)+G\left(\xi_{t+1}-E_{t} \xi_{t+1}\right),
$$

where the time dummy can be interpreted as an estimate of the inflation target for each period.

\subsubsection{Empirical findings}

To explore the empirical implications of this regional approach, we analyze semiannual CPI inflation and unemployment data from 27 MSAs in the United States. In our regressions we define $u_{t}(j)$ to be the period $t$ unemployment rate for MSA $j$, 
and $\pi_{t+1}(j)$ to be the inflation rate over the following year (i.e., $\left.C P I_{t+2}(j) / C P I_{t}(j)\right)$. Appendix $\mathrm{C}$ describes the data set in detail.

The stochastic component in equations (4) to (6) can potentially be correlated with the variables on the right-hand side to the extent that the shock $\varepsilon_{t+1}(j)$ can be autocorrelated. In that case, since $\varepsilon_{t}(j)$ will be correlated with $\pi_{t}(j)$ and $u_{t}(j)$, it may be important to use instrumental variables. Thus, we will report ordinary least squares (OLS) and two-stage least-squares (2SLS) results in what follows, where lagged values of the unemployment rate are used in the first stage.

Table 1 provides estimates for the coefficient $C$ in regressions (4) to (6) for four different subperiods of our sample. For these regressions, we used headline CPI inflation. The first three lines provide the OLS results, and the last three provide the 2SLS results using lagged unemployment rates as the instrument.

\begin{tabular}{|c|c||c|c|c|c|}
\hline \multicolumn{5}{|c|}{ Table 1: Estimates of coefficient $C$ in regional regressions (headline CPI) } \\
\hline Model & & $1977-1984$ & $1985-1990$ & $1991-2000$ & $2001-2010$ \\
\hline 4 & OLS & -0.03 & -0.26 & $-0.28^{* *}$ & $-0.20^{*}$ \\
\hline 5 & OLS & -0.08 & $-0.35^{*}$ & $-0.20^{*}$ & -0.26 \\
\hline 6 & OLS & $-0.31^{* *}$ & $-0.41^{* *}$ & $-0.34^{* *}$ & $-0.24^{* *}$ \\
\hline & & & & & -0.20 \\
\hline 4 & 2 SLS & 0.20 & -0.14 & $-0.43^{* *}$ & -0.24 \\
\hline 5 & 2 SLS & -0.02 & -0.49 & $-0.36^{* *}$ & $-0.22^{* *}$ \\
\hline 6 & 2 SLS & $-0.39^{* *}$ & $-0.29^{* *}$ & $-0.48^{* *}$ & \\
\hline \multirow{2}{*}{ *significant at the 5 percent level } \\
$* *$ significant at the 1 percent level
\end{tabular}

The results are striking. Except for the subperiod 1977-1984, the $C$ coefficients across models and periods are remarkably stable. During the final two periods, the 1990 s and $2000 \mathrm{~s}, C$ takes values between -0.20 and -0.48 . The outlier results 


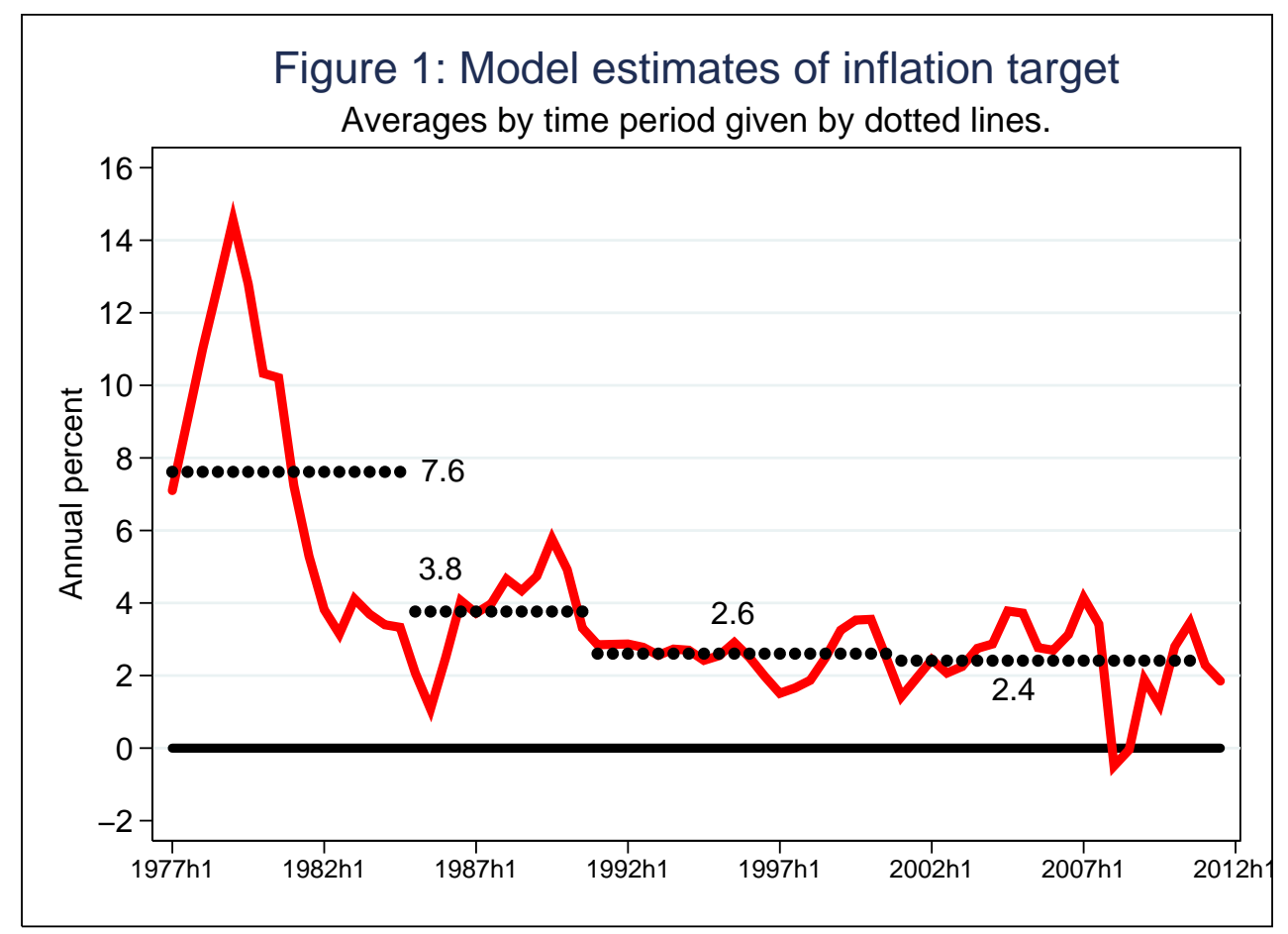

FiG. 1.

for the 1977-1984 period in two versions of the regressions are not surprising, since the assumption of a constant inflation target or an inflation target with bands is implausible given the behavior of inflation over that period. Those assumptions are much more plausible over the final two or three periods, and in those periods $C$ is surprisingly stable at around -0.3 . The time dummies in the third model can be interpreted as providing a period-by-period estimate of the inflation target which fluctuates over time, allowing for fluctuating inflation target results in remarkably stable estimates of $C$, including the first period.

In Figure 1, we plot the time dummies of regression (6). Not surprisingly, the pattern of the time dummies suggests that the assumption of constant inflation target (4) or a target with bands (5) does not fit well with the first subperiod.

Table 2 shows the results using core CPI inflation. Data for core inflation at the 
MSA level are available only for the last three periods. Here, the $C$ coefficients in the final three periods fall within an even tighter range, with values between -0.22 and -0.45 .

\begin{tabular}{|c|c|c|c|c|}
\hline \multicolumn{5}{|c|}{ Table 2: Estimates of coefficient $C$ in regional regressions (core CPI) } \\
\hline Model & & $1985-1990$ & $1991-2000$ & $2001-2010$ \\
\hline 4 & OLS & $-0.421^{* *}$ & $-0.39^{* *}$ & $-0.30^{* *}$ \\
\hline 5 & OLS & $-0.42^{* *}$ & $-0.22^{*}$ & $-0.34^{* *}$ \\
\hline 6 & OLS & $-0.45^{* *}$ & $-0.38^{* *}$ & $-0.34^{* *}$ \\
\hline & & & & $-0.23^{*}$ \\
\hline 4 & 2 SLS & -0.27 & $-0.44^{* *}$ & $-0.26^{*}$ \\
\hline 5 & 2 SLS & $-0.34^{*}$ & $-0.29^{*}$ & $-0.26^{* *}$ \\
\hline 6 & 2 SLS & $-0.29^{*}$ & $-0.43^{* *}$ & \\
\hline \multirow{2}{*}{$\begin{array}{l}\text { * significant at the } 5 \text { percent level } \\
* * \text { significant at the } 1 \text { percent level }\end{array}$} \\
\hline
\end{tabular}

In summary, the coefficient $C$ is remarkably stable around -0.3 .

\section{The Dual Mandate Model}

The literature has clearly documented that a nonnegative coefficient can be estimated in Phillips curve regressions, at least for some subperiods of recent U.S. history. ${ }^{11}$ Those results contradict our simple theory, which predicts those coefficients to be zero.

As we show in the next subsection, a more general model of the central bank that allows for regime changes is fully consistent with those results while leaving unchanged the conclusions reached in the previous section on the stability of the $C$ coefficient using regional regressions.

\footnotetext{
${ }^{11}$ Atkeson and Ohanian (2001) and Fisher, Liu, and Zhou (2002).
} 
Consider the same underlying model but imagine now that the central bank acts according to a dual mandate. In particular, imagine it solves the following policy problem:

$$
\min _{i_{t}} \frac{1}{2} E_{t}\left\{\gamma\left[\pi_{t+1}-\pi_{t+1}^{*}\right]^{2}+(1-\gamma)\left[u_{t+1}-u_{t+1}^{*}\right]^{2}\right\}
$$

given the solutions (1) and (2), where and $\left\{\pi_{t+1}^{*}, u_{t+1}^{*}\right\}$ are the time-varying targets for inflation and unemployment and $\gamma$ is the weight the central bank puts on its inflation target. We assume this weight to be time invariant, but as we will see, the data suggest that it has changed across subperiods.

The solution for the optimal policy is given by ${ }^{12}$

$$
i_{t}=\Pi_{t}-\frac{\left[\gamma F B+(1-\gamma) F^{\prime} B^{\prime}\right]}{\left[\gamma F^{2}+(1-\gamma) F^{\prime 2}\right]} \pi_{t}-\frac{\left[\gamma F C+(1-\gamma) F^{\prime} C^{\prime}\right]}{\left[\gamma F^{2}+(1-\gamma) F^{\prime 2}\right]} u_{t}
$$

where

$$
\Pi_{t}=\frac{\gamma F \pi_{t+1}^{*}+(1-\gamma) F^{\prime} u_{t+1}^{*}-\left(\gamma F A+(1-\gamma) F^{\prime} A^{\prime}\right)-\left[\gamma F G+(1-\gamma) F^{\prime} G^{\prime}\right] E_{t} \xi_{t+1}}{\left[\gamma F^{2}+(1-\gamma) F^{\prime 2}\right]}
$$

Replacing the solution for inflation,

$$
\begin{aligned}
\pi_{t+1}= & A-F \frac{\gamma F A+(1-\gamma) F^{\prime} A^{\prime}}{\left[\gamma F^{2}+(1-\gamma) F^{2}\right]}-\frac{F\left[\gamma F G+(1-\gamma) F^{\prime} G^{\prime}\right]}{\left[\gamma F^{2}+(1-\gamma) F^{2}\right]} E_{t} \xi_{t+1} \\
& +\left(B-F \frac{\left[\gamma F B+(1-\gamma) F^{\prime} B^{\prime}\right]}{\left[\gamma F^{2}+(1-\gamma) F^{\prime 2}\right]}\right) \pi_{t}+\left(C-F \frac{\left[\gamma F C+(1-\gamma) F^{\prime} C^{\prime}\right]}{\left[\gamma F^{2}+(1-\gamma) F^{\prime 2}\right]}\right) u_{t} \\
& +\frac{F^{2} \gamma}{\left[\gamma F^{2}+(1-\gamma) F^{\prime 2}\right]} \pi_{t+1}^{*}+\frac{F F^{\prime}(1-\gamma)}{\left[\gamma F^{2}+(1-\gamma) F^{\prime 2}\right]} u_{t+1}^{*} .
\end{aligned}
$$

Thus, the marginal effect of unemployment on future inflation is given by the coeffi-

cient

$$
\left(C-F \frac{\left[\gamma F C+(1-\gamma) F^{\prime} C^{\prime}\right]}{\left[\gamma F^{2}+(1-\gamma) F^{\prime 2}\right]}\right)
$$

which depends on the policy parameter $\gamma$. In general, if $\gamma \in(0,1)$, the coefficient may take any value, depending on the values for $F, F^{\prime}, C, C$.

\footnotetext{
${ }^{12}$ The details are in Appendix A.
} 
Note also that if there are shifts over time in the weight the central bank assigns to each target, the estimated coefficient would be time varying, as the empirical literature has found. ${ }^{13}$

If $\gamma=1$, the coefficient becomes

$$
\left(C-\frac{\left[\gamma F C+(1-\gamma) F^{\prime} C^{\prime}\right]}{\left[\gamma F^{2}+(1-\gamma) F^{\prime 2}\right]}\right)=\left(C-F \frac{[\gamma F C]}{\gamma F^{2}}\right)=C-C=0
$$

as in the single mandate model.

\subsection{The Regional Equations}

We now analyze the equilibrium solution for each of the regions. The solution for the model is

$$
\pi_{t+1}(j)=A+B \pi_{t}(j)+C u_{t}(j)+F i_{t}+G\left(\varepsilon_{t+1}(j)+\xi_{t+1}\right),
$$

and the policy rate is

$$
i_{t}^{O p t}=\Pi_{t}-\frac{\left[\gamma F B+(1-\gamma) F^{\prime} B^{\prime}\right]}{\left[\gamma F^{2}+(1-\gamma) F^{\prime 2}\right]} \pi_{t}-\frac{\left[\gamma F C+(1-\gamma) F^{\prime} C^{\prime}\right]}{\left[\gamma F^{2}+(1-\gamma) F^{\prime 2}\right]} u_{t}
$$

Replacing $i_{t}$ with $i_{t}^{\text {Opt }}$ we obtain

$$
\begin{aligned}
\pi_{t+1}(j)= & {\left[A+F \Pi_{t}\right]+B \pi_{t}(j)+C u_{t}(j)+F A_{t}+G\left(\varepsilon_{t+1}(j)+\xi_{t+1}\right) } \\
& -F \frac{\left[\gamma F B+(1-\gamma) F^{\prime} B^{\prime}\right]}{\left[\gamma F^{2}+(1-\gamma) F^{\prime 2}\right]} \pi_{t}-F \frac{\left[\gamma F C+(1-\gamma) F^{\prime} C^{\prime}\right]}{\left[\gamma F^{2}+(1-\gamma) F^{\prime 2}\right]} u_{t} .
\end{aligned}
$$

This equation is different from (3) in two important respects. First the coefficient on regional unemployment is the one that provides information regarding the structural parameter $C$, whereas the coefficient on average unemployment is no longer its negative. Thus, both regional and national unemployment should appear independently

\footnotetext{
${ }^{13} \mathrm{~A}$ change in $\gamma$ could be interpreted as a regime shift, since it would affect the policy rule and the equilibrium of the model. A regime shift of this type is also consistent with the analysis in Sargent (1999).
} 
on the right-hand side of the regression (the same applies to regional and national inflation). Second, the variable $\Pi_{t}$ is a nonlinear function of the two policy targets and the structural parameters of the model. To the extent that the targets are constant over time, so will be the term $\left[A+F \Pi_{t}\right]$, in which case adding a constant to the regression should suffice and a regression similar to (4) can be estimated. Similarly, a time dummy can certainly be interpreted as an estimate of $\left[A+F \Pi_{t}\right]$, since the term does not involve any region-specific information. Note, finally, that there is no counterpart to (5) in this case.

We now repeat the regressions (4) and (6) we did in Section 2, but this time we allow for both $u_{t}(j)$ and $u_{t}$ to enter independently on the right-hand side, rather than using the difference between the two. The results are reported in Tables 3 and 4 for headline and core inflation, respectively.

\begin{tabular}{|c|c|c|c||c|c|c|c|c|c|c|c|}
\hline \multicolumn{8}{|c|}{ Table 3: Estimates of unemployment coefficients in regional regressions (headline CPI) } \\
\hline Model & & \multicolumn{2}{|c||}{$1977-2010$} & $1977-1984$ & \multicolumn{2}{c|}{$1985-1990$} & \multicolumn{2}{c|}{$1991-2000$} & \multicolumn{2}{c|}{$2001-2010$} \\
\hline & & $u_{t}$ & $u_{t}(j)$ & $u_{t}$ & $u_{t}(j)$ & $u_{t}$ & $u_{t}(j)$ & $u_{t}$ & $u_{t}(j)$ & $u_{t}$ & $u_{t}(j)$ \\
\hline 4 & OLS & $0.17^{* *}$ & $-0.21^{* *}$ & $-1.43^{* *}$ & $-0.25^{*}$ & $-1.36^{* *}$ & $-0.45^{* *}$ & $0.23^{* *}$ & $-0.28^{* *}$ & -0.01 & $-0.24^{* *}$ \\
\hline 6 & OLS & $1.18^{* *}$ & $-0.30^{* *}$ & -0.32 & $-0.31^{* *}$ & $-1.29^{* *}$ & $-0.41^{* *}$ & $.42^{* *}$ & $-0.34^{* *}$ & $0.55^{* *}$ & $-0.24^{* *}$ \\
\hline 4 & 2 SLS & $0.36^{* *}$ & -0.16 & $-5.33^{* *}$ & -0.85 & $-1.38^{* *}$ & $-0.41^{*}$ & $0.35^{* *}$ & $-0.46^{* *}$ & $0.51^{* *}$ & -0.14 \\
\hline 6 & 2 SLS & $0.85^{* *}$ & $-0.31^{* *}$ & $1.02^{* *}$ & $-0.39^{* *}$ & $-0.95^{* *}$ & $-0.29^{* *}$ & $0.54^{* *}$ & $-0.48^{* *}$ & $0.96^{* *}$ & $-0.22^{* *}$ \\
\hline
\end{tabular}




\begin{tabular}{|c|c|c|c||c|c|c|c|c|c|c|c|}
\hline \multicolumn{9}{|c|}{ Table 4: Estimates of unemployment coefficients in regional regressions (core CPI) } \\
\hline Model & & $1984-2010$ & $1977-1984$ & $1985-1990$ & \multicolumn{2}{c|}{$1991-2000$} & \multicolumn{2}{c|}{$2001-2010$} \\
\hline & & $u_{t}$ & $u_{t}(j)$ & $u_{t}$ & $u_{t}(j)$ & $u_{t}$ & $u_{t}(j)$ & $u_{t}$ & $u_{t}(j)$ & $u_{t}$ & $u_{t}(j)$ \\
\hline 4 & OLS & $0.28^{* *}$ & $-0.37^{* *}$ & & & -0.00 & $-0.47^{* *}$ & $0.28^{* *}$ & $-0.32^{* *}$ & 0.08 & $-0.36^{* *}$ \\
\hline 6 & OLS & $0.24^{* *}$ & $-0.35^{* *}$ & & & -0.09 & $-0.45^{* *}$ & $0.29^{* *}$ & $-0.38^{* *}$ & 0.07 & $-0.34^{* *}$ \\
\hline & & & & & & & & & & & \\
\hline 4 & 2 SLS & $0.36^{* *}$ & $-0.31^{* *}$ & & & -0.12 & $-0.39^{* *}$ & $0.33^{*}$ & $-0.39^{* *}$ & $0.57^{* *}$ & -0.18 \\
\hline 6 & 2 SLS & $0.28^{* *}$ & $-0.29^{* *}$ & & & -0.23 & $-0.29^{*}$ & $0.35^{* *}$ & $-0.43^{* *}$ & $-0.33^{* *}$ & $-0.26^{* *}$ \\
\hline
\end{tabular}

The first notable result is that the coefficients on the regional unemployment rate in these dual mandate regressions again remain remarkably near -0.3 for each of the subperiods. Even under the assumption of a constant target, the coefficient on $u_{t}(j)$ is estimated to be close to -0.3 for the first subsample; it is -0.25 versus -0.03 in Table 1. The estimated value for the 2SLS version becomes too negative, but the standard error is large.

Another interesting feature of the results is that one has the beginning of a theory that explains why the unemployment coefficient in regressions that use aggregate data changes over time in response to changes in policy regimes. For example, the assumption of pure inflation target $\gamma=1$ is consistent with the results for the 1990s. This is the case in which the coefficient on national unemployment is equal to minus the coefficient on regional unemployment. In the case of CPI headline, this is clearly off the mark for the first two subsamples but remarkably close for the third. A similar, though less drastic, pattern holds for core inflation. The pattern changes for the last subperiod. ${ }^{14}$ This is consistent with the hypothesis that $\gamma$ is lower than 1 at the

\footnotetext{
${ }^{14}$ One might think that monetary policy after the 2008 crisis implied a regime change, and that
} 
beginning and becomes close to 1 by the 1990s. In turn, this finding is consistent with the analysis in Sargent (1999) that argues that by the 1990s, the central bank was essentially targeting inflation — much unlike its behavior in the 1970s.

\section{Conclusions}

The literature on the stability of NAIRU-type Phillips curves has ignored the impact of endogenous monetary policy on Phillips curve regression coefficients. We argue that this omission has important implications: when policy is endogenous, regressions on aggregate data are uninformative as to the existence of a stable relationship between unemployment and future inflation. We provide a simple framework that incorporates endogenous policy. We show that if the NAIRU model is assumed to be true, regional data can be used to identify the structural relationship between unemployment and inflation. Using data from 1976 to 2010 and from 27 MSAs, our analysis cannot reject the hypothesis that a 1 percentage point increase in the unemployment rate is associated with a roughly 0.3 percentage point decline in inflation over the next year. This holds over the entire time period and, more remarkably, over each of the four subperiods for the model with a time-varying inflation target.

We caution that these results should not be interpreted as providing conclusive evidence of a stable Phillips curve, because the findings may well be consistent with other interpretations. One could consider alternative theoretical frameworks in line with the literature that studies movements in real exchange rates in open economies. Indeed, if there are goods that are not traded across cities, differences in regional inflation rates reflect, at least partially, movements in relative prices that are different across cities. Imagine a demand shock for some of those nontraded goods in a particular city. The resulting increase in production would reduce unemployment and would explains why the results for this subperiod differ from the $\gamma=1$ case. However, when we repeated the regressions up until 2007, the results also differ. 
increase the relative price of the nontraded good, creating a negative relationship between unemployment and inflation. ${ }^{15}$ With relatively rich dynamics, that alternative theoretical framework will most likely be consistent with our regional regressions as well.

\footnotetext{
${ }^{15}$ We repeated the regressions but used inflation in services first and then in goods, and the coefficients for the services inflation regression were higher in absolute value.
} 


\section{Appendix A}

\section{A Solution for the Optimal Policy}

The problem is

$$
\min _{i_{t}} \frac{1}{2} E_{t}\left\{\gamma\left[\pi_{t+1}-\pi_{t+1}^{*}\right]^{2}+(1-\gamma)\left[\pi_{t+1}-\pi_{t+1}^{*}\right]^{2}\right\}
$$

given (1) and (2). Replacing the constraints, we obtain

$$
\begin{aligned}
& \min _{i_{t}} \frac{1}{2} E_{t}\left\{\gamma\left[A+B \pi_{t}+C u_{t}+F i_{t}+G \xi_{t+1}-\pi_{t+1}^{*}\right]^{2}\right. \\
& \left.+(1-\gamma)\left[A^{\prime}+B^{\prime} \pi_{t}+C^{\prime} u_{t}+F^{\prime} i_{t}+G^{\prime} \xi_{t+1}-u_{t+1}^{*}\right]^{2}\right\} .
\end{aligned}
$$

The solution is

$$
\begin{aligned}
0= & E_{t}\left\{\gamma F\left[A+B \pi_{t}+C u_{t}+F i_{t}+G \xi_{t+1}-\pi_{t+1}^{*}\right]\right. \\
& \left.+(1-\gamma) F^{\prime}\left[A^{\prime}+B^{\prime} \pi_{t}+C^{\prime} u_{t}+F^{\prime} i_{t}^{O p t}+G^{\prime} \xi_{t+1}-u_{t+1}^{*}\right]\right\} .
\end{aligned}
$$

Taking expectations, we obtain

$$
\begin{aligned}
0= & \gamma F\left[A+B \pi_{t}+C u_{t}+F i_{t}+E_{t} G \xi_{t+1}-\pi_{t+1}^{*}\right] \\
& +(1-\gamma) F^{\prime}\left[A^{\prime}+B^{\prime} \pi_{t}+C^{\prime} u_{t}+F^{\prime} i_{t}^{O p t}+E_{t} G^{\prime} \xi_{t+1}-u_{t+1}^{*}\right],
\end{aligned}
$$

since the targets are known at time $t$. This can be written as

$$
\begin{aligned}
0= & \gamma F A+(1-\gamma) F^{\prime} A^{\prime}+\left[\gamma F B+(1-\gamma) F^{\prime} B^{\prime}\right] \pi_{t}+\left[\gamma F C+(1-\gamma) F^{\prime} C^{\prime}\right] u_{t} \\
& +\left[\gamma F^{2}+(1-\gamma) F^{\prime 2}\right] i_{t}^{O p t}+\left[\gamma F G+(1-\gamma) F^{\prime} G^{\prime}\right] E_{t} \xi_{t+1}-\left[\gamma F \pi_{t+1}^{*}+(1-\gamma) F^{\prime} u_{t+1}^{*}\right] .
\end{aligned}
$$

The solution is given by

$$
i_{t}^{O p t}=\Pi_{t}-\frac{\left[\gamma F B+(1-\gamma) F^{\prime} B^{\prime}\right] \pi_{t}+\left[\gamma F C+(1-\gamma) F^{\prime} C^{\prime}\right] u_{t}}{\left[\gamma F^{2}+(1-\gamma) F^{\prime 2}\right]},
$$

where

$$
\Pi_{t}=\frac{\gamma F \pi_{t+1}^{*}+(1-\gamma) F^{\prime} u_{t+1}^{*}-\left[\gamma F G+(1-\gamma) F^{\prime} G^{\prime}\right] E_{t} \xi_{t+1}-\gamma F A+(1-\gamma) F^{\prime} A^{\prime}}{\left[\gamma F^{2}+(1-\gamma) F^{\prime 2}\right]} .
$$


Replacing the solution for inflation, we have

$$
\begin{aligned}
\pi_{t+1}= & A+\left[B-F \frac{\left[\gamma F B+(1-\gamma) F^{\prime} B^{\prime}\right]}{\left[\gamma F^{2}+(1-\gamma) F^{\prime 2}\right]}\right] \pi_{t} \\
& +\left[C-F \frac{\left[\gamma F C+(1-\gamma) F^{\prime} C^{\prime}\right]}{\left[\gamma F^{2}+(1-\gamma) F^{\prime 2}\right]}\right] u_{t}+ \\
& F \frac{\gamma F \pi_{t+1}^{*}+(1-\gamma) F^{\prime} u_{t+1}^{*}-\gamma F A+(1-\gamma) F^{\prime} A^{\prime}}{\left[\gamma F^{2}+(1-\gamma) F^{\prime 2}\right]} \\
& -F \frac{\left[\gamma F G+(1-\gamma) F^{\prime} G^{\prime}\right] E_{t} \xi_{t+1}}{\left[\gamma F^{2}+(1-\gamma) F^{\prime 2}\right]}+G \xi_{t+1}
\end{aligned}
$$

or

$$
\begin{aligned}
\pi_{t+1}= & A-F \frac{\gamma F A+(1-\gamma) F^{\prime} A^{\prime}}{\left[\gamma F^{2}+(1-\gamma) F^{\prime 2}\right]} \\
& +\left(B-F \frac{\left[\gamma F B+(1-\gamma) F^{\prime} B^{\prime}\right]}{\left[\gamma F^{2}+(1-\gamma) F^{\prime 2}\right]}\right) \pi_{t} \\
& +\left(C-F \frac{\left[\gamma F C+(1-\gamma) F^{\prime} C^{\prime}\right]}{\left[\gamma F^{2}+(1-\gamma) F^{\prime 2}\right]}\right) u_{t} \\
& +\frac{F^{2} \gamma}{\left[\gamma F^{2}+(1-\gamma) F^{\prime 2}\right]} \pi_{t+1}^{*}+\frac{F F^{\prime}(1-\gamma)}{\left[\gamma F^{2}+(1-\gamma) F^{\prime 2}\right]} u_{t+1}^{*} \\
& -\frac{F\left[\gamma F G+(1-\gamma) F^{\prime} G^{\prime}\right]}{\left[\gamma F^{2}+(1-\gamma) F^{\prime 2}\right]} E_{t} \xi_{t+1}+G \xi_{t+1} .
\end{aligned}
$$

\section{A.1 The effect on the regional equations}

The solution for the model is

$$
\pi_{t+1}(j)=A+B \pi_{t}(j)+C u_{t}(j)+F i_{t}+G\left(\varepsilon_{t+1}(j)+\xi_{t+1}\right),
$$

and the policy rate is

$$
i_{t}^{O p t}=\Pi_{t}-\frac{\left[\gamma F B+(1-\gamma) F^{\prime} B^{\prime}\right]}{\left[\gamma F^{2}+(1-\gamma) F^{\prime 2}\right]} \pi_{t}-\frac{\left[\gamma F C+(1-\gamma) F^{\prime} C^{\prime}\right]}{\left[\gamma F^{2}+(1-\gamma) F^{\prime 2}\right]} u_{t},
$$

where

$$
\Pi_{t}=\frac{\gamma F \pi_{t+1}^{*}+(1-\gamma) F^{\prime} u_{t+1}^{*}-\left(+\gamma F A+(1-\gamma) F^{\prime} A^{\prime}\right)-\left[\gamma F G+(1-\gamma) F^{\prime} G^{\prime}\right] E_{t} \xi_{t+1}}{\left[\gamma F^{2}+(1-\gamma) F^{\prime 2}\right]} .
$$


Some algebra gives us

$$
\begin{aligned}
\pi_{t+1}(j)= & A+B \pi_{t}(j)+C u_{t}(j)+G\left(\varepsilon_{t+1}(j)+\xi_{t+1}\right) \\
& +F\left(\Pi_{t}-\frac{\left[\gamma F B+(1-\gamma) F^{\prime} B^{\prime}\right]}{\left[\gamma F^{2}+(1-\gamma) F^{\prime 2}\right]} \pi_{t}-\frac{\left[\gamma F C+(1-\gamma) F^{\prime} C^{\prime}\right]}{\left[\gamma F^{2}+(1-\gamma) F^{\prime 2}\right]} u_{t}\right) .
\end{aligned}
$$




\section{Appendix B}

\section{B An Old Keynesian Model}

In this appendix we present a slightly modified version of the model used by Taylor (1999) and discussed in Cochrane (2011). The model specifies a NAIRU-type Phillips curve, where the growth rate of inflation holds a negative linear relationship with the difference between the current unemployment rate and a constant level (known as the natural rate of unemployment). Thus, we write

$$
\pi_{t}-\pi_{t-1}=-\gamma\left(u_{t-1}-u\right)-\varepsilon\left(u_{t}-u\right)+e_{t}^{\pi},
$$

where $\pi_{t}$ is the inflation rate, $u_{t}$ is the unemployment rate, $\gamma, u, \varepsilon$ are positive parameters, and $e_{t}^{\pi}$ is a shock. This is the same equation used by Taylor (1999), except that he assumes $\varepsilon=0 .{ }^{16}$ This assumption implies that the policy rate has no immediate effect on the inflation rate. By letting $\varepsilon>0$, albeit it is small, we allow for that immediate effect.

The second equation establishes a negative linear relationship between unemployment and the difference between the policy interest rate and the inflation rate, so we write

$$
u_{t}=\sigma\left(i_{t}-\pi_{t}-r\right)+e_{t}^{u},
$$

where $\sigma, r$ are positive parameters and $e_{t}^{u}$ is a shock. ${ }^{17}$

\footnotetext{
${ }^{16}$ Taylor's model is expressed in terms of output deviations instead of unemployment deviations. Our specification implies a negative linear relationship between output deviations and unemployment deviations.

${ }^{17}$ To the extent that the term in parentheses on the right-hand side of this equation aims at capturing movements in the real interest rate as deviations from $r$ (presumably its steady state value), the fact that $\pi_{t}$ rather than $E_{t} \pi_{t+1}$ is in this equation may appear surprising. However, as
} 
In what follows, we interpret the unemployment rate as deviations from its steady state level $u$, or, equivalently, we set $u=0$.

Using the second in the first, we have

$$
\pi_{t}=\pi_{t-1}-\gamma u_{t-1}-\varepsilon\left(\sigma\left(i_{t}-\pi_{t}-r\right)+e_{t}^{u}\right)+e_{t}^{\pi}
$$

or

$$
\pi_{t}=\frac{\pi_{t-1}-\gamma u_{t-1}+\sigma \varepsilon r-\sigma \varepsilon i_{t}-\varepsilon e_{t}^{u}+e_{t}^{\pi}}{(1-\varepsilon \sigma)},
$$

whereas using the first in the second, we have

$$
\begin{gathered}
u_{t}(1-\varepsilon \sigma)=\sigma\left(i_{t}-\pi_{t-1}+\gamma u_{t-1}-e_{t}^{\pi}-r\right)+e_{t}^{u} \\
u_{t}=-\frac{\sigma}{(1-\sigma \varepsilon)} \pi_{t-1}+\frac{\sigma \gamma}{(1-\sigma \varepsilon)} u_{t-1}+\frac{\sigma i_{t}-\sigma r-\sigma e_{t}^{\pi}+e_{t}^{u}}{(1-\sigma \varepsilon)} .
\end{gathered}
$$

Thus, we can write the system as

$$
\begin{aligned}
{\left[\begin{array}{l}
\pi_{t} \\
u_{t}
\end{array}\right]=} & {\left[\begin{array}{cc}
\frac{1}{(1-\varepsilon \sigma)} & -\frac{\gamma}{(1-\varepsilon \sigma)} \\
-\frac{\sigma}{(1-\sigma \varepsilon)} & \frac{\sigma \gamma}{(1-\sigma \varepsilon)}
\end{array}\right]\left[\begin{array}{l}
\pi_{t-1} \\
u_{t-1}
\end{array}\right]+\left[\begin{array}{c}
-\frac{\sigma \varepsilon}{(1-\varepsilon \sigma)} \\
\frac{\sigma}{(1-\varepsilon \sigma)}
\end{array}\right]\left(i_{t}-r\right)+} \\
& {\left[\begin{array}{cc}
-\frac{\varepsilon}{(1-\varepsilon \sigma)} & \frac{1}{(1-\varepsilon \sigma)} \\
\frac{1}{(1-\sigma \varepsilon)} & -\frac{\sigma}{(1-\sigma \varepsilon)}
\end{array}\right]\left[\begin{array}{c}
e_{t}^{u} \\
e_{t}^{\pi}
\end{array}\right] }
\end{aligned}
$$

\section{B.1 The interest rate rule}

If we assume, as Taylor (1999) and Cochrane (2011) do, that

$$
i_{t}=r+\phi_{\pi} \pi_{t}+\phi_{y} y_{t}
$$

we show below, this equation - with a reinterpretation of the parameters - will arise exactly as the solution in any case, as long as $\varepsilon$ is zero. Given the lack of microfoundations, this reinterpretation seems innocuous to us. 
then the solution is

$$
\begin{aligned}
{\left[\begin{array}{c}
\pi_{t} \\
u_{t}
\end{array}\right]=} & {\left[\begin{array}{cc}
\frac{\left(1+\sigma \phi_{u}\right)}{\left(1+\sigma \phi_{u}\right)+\sigma\left(\phi_{\pi}-1\right) \varepsilon} & -\frac{\left(1+\sigma \phi_{u}\right)}{\left(1+\sigma \phi_{u}\right)+\sigma\left(\phi_{\pi}-1\right) \varepsilon} \gamma \\
\frac{\sigma\left(\phi_{\pi}-1\right)}{\left(1+\sigma \phi_{u}\right)+\sigma\left(\phi_{\pi}-1\right) \varepsilon} & -\frac{\sigma\left(\phi_{\pi}-1\right)}{\left(1+\sigma \phi_{u}\right)+\sigma\left(\phi_{\pi}-1\right) \varepsilon} \gamma
\end{array}\right]\left[\begin{array}{c}
\pi_{t-1} \\
u_{t-1}
\end{array}\right]+} \\
& \frac{1}{\left(1+\sigma \phi_{u}\right)+\sigma\left(\phi_{\pi}-1\right) \varepsilon}\left[\begin{array}{cc}
-\varepsilon & 1 \\
1 & \sigma\left(\phi_{\pi}-1\right)
\end{array}\right]\left[\begin{array}{c}
e_{t}^{u} \\
e_{t}^{\pi}
\end{array}\right]
\end{aligned}
$$

The two roots are given by

$$
\begin{aligned}
\lambda_{1} \lambda_{2} & =0 \\
\lambda_{1}+\lambda_{2} & =\frac{\left(1+\sigma \phi_{u}\right)-\left(\phi_{\pi}-1\right) \sigma \gamma}{\left(1+\sigma \phi_{u}\right)+\sigma\left(\phi_{\pi}-1\right) \varepsilon}
\end{aligned}
$$

so one root is zero, and the other is given by

$$
\frac{\left(1+\sigma \phi_{u}\right)-\left(\phi_{\pi}-1\right) \sigma \gamma}{\left(1+\sigma \phi_{u}\right)+\sigma\left(\phi_{\pi}-1\right) \varepsilon}
$$

which is less than one as long as $\phi_{\pi}>1$, as described in Taylor (1999). Therefore, the system has a unique bounded solution.

\section{B.2 Characterizing the optimal policy rule}

Recall that the solution is given by

$$
\begin{aligned}
{\left[\begin{array}{l}
\pi_{t} \\
u_{t}
\end{array}\right]=} & {\left[\begin{array}{cc}
\frac{1}{(1-\varepsilon \sigma)} & -\frac{\gamma}{(1-\varepsilon \sigma)} \\
-\frac{\sigma}{(1-\sigma \varepsilon)} & \frac{\sigma \gamma}{(1-\sigma \varepsilon)}
\end{array}\right]\left[\begin{array}{l}
\pi_{t-1} \\
u_{t-1}
\end{array}\right]+\left[\begin{array}{c}
-\frac{\sigma \varepsilon}{(1-\varepsilon \sigma)} \\
\frac{\sigma}{(1-\varepsilon \sigma)}
\end{array}\right]\left(i_{t}-r\right)+} \\
& {\left[\begin{array}{cc}
-\frac{\varepsilon}{(1-\varepsilon \sigma)} & \frac{1}{(1-\varepsilon \sigma)} \\
\frac{1}{(1-\sigma \varepsilon)} & -\frac{\sigma}{(1-\sigma \varepsilon)}
\end{array}\right]\left[\begin{array}{c}
e_{t}^{u} \\
e_{t}^{\pi}
\end{array}\right], }
\end{aligned}
$$

so, in the notation of the paper,

$$
\pi_{t+1}=A+B \pi_{t}+C u_{t}+F i_{t}+\xi_{t}^{\pi},
$$

So

$$
B=\frac{1}{(1-\varepsilon \sigma)}, C=-\frac{\gamma}{(1-\varepsilon \sigma)}, F=-\frac{\sigma \varepsilon}{(1-\varepsilon \sigma)}
$$


and the optimal policy is

$$
i_{t}^{O p t}=\frac{1}{F}\left[\pi_{t+1}^{*}-\left(A+B \pi_{t}+C u_{t}+E_{t} \xi_{t+1}^{\pi}\right)\right]
$$

SO

$$
i_{t}^{O p t}=\frac{1}{-\frac{\sigma \varepsilon}{(1-\varepsilon \sigma)}}\left[\pi_{t+1}^{*}-\left(A+\frac{1}{(1-\varepsilon \sigma)} \pi_{t}+-\frac{\gamma}{(1-\varepsilon \sigma)} u_{t}+E_{t} \xi_{t+1}^{\pi}\right)\right]
$$

or

$$
i_{t}^{O p t}=\left[-\frac{(1-\varepsilon \sigma)}{\sigma \varepsilon} \pi_{t+1}^{*}+\frac{(1-\varepsilon \sigma)}{\sigma \varepsilon} A+\frac{1}{\sigma \varepsilon} \pi_{t}-\frac{\gamma}{\sigma \varepsilon} u_{t}-E_{t} \xi_{t+1}^{\pi}\right] .
$$

Thus, as long as $\sigma \varepsilon<1$, which will hold for small values of $\varepsilon$, the conditions for a unique stable solution are satisfied.

\section{B.3 The reduced form parameter versus the structural form parameter}

The solution of the model is given by

$$
\begin{aligned}
{\left[\begin{array}{c}
\pi_{t} \\
u_{t}
\end{array}\right]=} & {\left[\begin{array}{cc}
\frac{\left(1+\sigma \phi_{u}\right)}{\left(1+\sigma \phi_{u}\right)+\sigma\left(\phi_{\pi}-1\right) \varepsilon} & -\frac{\left(1+\sigma \phi_{u}\right)}{\left(1+\sigma \phi_{u}\right)+\sigma\left(\phi_{\pi}-1\right) \varepsilon} \gamma \\
\frac{\sigma\left(\phi_{\pi}-1\right)}{\left(1+\sigma \phi_{u}\right)+\sigma\left(\phi_{\pi}-1\right) \varepsilon} & -\frac{\sigma\left(\phi_{\pi}-1\right)}{\left(1+\sigma \phi_{u}\right)+\sigma\left(\phi_{\pi}-1\right) \varepsilon} \gamma
\end{array}\right]\left[\begin{array}{c}
\pi_{t-1} \\
u_{t-1}
\end{array}\right]+} \\
& \frac{1}{\left(1+\sigma \phi_{u}\right)+\sigma\left(\phi_{\pi}-1\right) \varepsilon}\left[\begin{array}{cc}
-\varepsilon & 1 \\
1 & \sigma\left(\phi_{\pi}-1\right)
\end{array}\right]\left[\begin{array}{c}
e_{t}^{u} \\
e_{t}^{\pi}
\end{array}\right],
\end{aligned}
$$

so we can write the solution for inflation as

$$
\pi_{t}=\frac{\left(1+\sigma \phi_{u}\right)}{\left(1+\sigma \phi_{u}\right)+\sigma\left(\phi_{\pi}-1\right) \varepsilon} \pi_{t-1}-\frac{\left(1+\sigma \phi_{u}\right)}{\left(1+\sigma \phi_{u}\right)+\sigma\left(\phi_{\pi}-1\right) \varepsilon} \gamma u_{t-1}+\frac{e_{t}^{\pi}-\varepsilon e_{t}^{u}}{\left(1+\sigma \phi_{u}\right)+\sigma\left(\phi_{\pi}-1\right) \varepsilon} .
$$

Thus, the reduced form parameter $\widehat{\gamma}$ is equal to

$$
\widehat{\gamma}=\frac{\left(1+\sigma \phi_{u}\right)}{\left(1+\sigma \phi_{u}\right)+\sigma\left(\phi_{\pi}-1\right) \varepsilon} \gamma=\frac{1}{1+\varepsilon \frac{\sigma\left(\phi_{\pi}-1\right)}{\left(1+\sigma \phi_{u}\right)}} \gamma
$$

so it is lower than the structural parameter $\gamma$, but arbitrarily close when $\varepsilon$ is close to zero. 


\section{Appendix C}

\section{Description of Data}

This appendix describes our data sources and calculations. We analyze semiannual CPI inflation and unemployment data for the United States and for 27 metropolitan statistical areas (MSAs). All semiannual data for unemployment and CPI price indices are computed as the arithmetic average of monthly data for the first and second half of each year. Inflation and price data for MSAs are only available as not seasonally adjusted, so all data are not seasonally adjusted.

\section{C.1 Inflation data.-}

The Bureau of Labor Statistics (BLS) publishes CPI data for 27 MSAs. The BLS publishes seminanual data for 13 MSAs, and higher frequency data (monthly or bimonthly) for the other 14 MSAs. We use semiannual data to obtain the largest possible sample. Headline CPI is available back to 1941 for 23 MSAs, with data for the remaining MSAs starting in 1977, 1987, 1997, and 2002. Core CPI is available back to 1982 for 24 MSAs, with data for the remaining MSAs starting in 1987, 1997, and 2002. When semiannual data is not available as a published series, we compute the semiannual average following BLS methodology: first, interpolate the missing monthly indices using a geometric mean of values in adjacent months; second, calculate the arithmetic average of the monthly data in the first and second half of each year.

\section{2 Unemployment data.-}

The BLS publishes monthly unemployment rate, not seasonally adjusted, for each of the 27 MSAs with corresponding CPI price indices. Published BLS data is available back to 1990. The BLS has unpublished unemployment data back to 1976, but these 
data are not consistent with the published data due to changes in the MSA geographic definitions and other factors. However, the BLS also has unemployment and labor force data by county going back to 1976 . We used the county level data to construct a geographically consistent definition of MSAs going back to 1976. The constructed unemployment and labor force series overlap very closely with the published data in the post 1990 period. We combine our pre-1990 constructed unemployment rates with the published data to obtain unemployment rate series back to 1976. The lack of readily accessible unemployment data prior to 1976 is a limiting data factor for our analysis. 


\section{References}

Atkeson, A., and L. Ohanian (2001): "Are Phillips Curves Useful for Forecasting Inflation?" Federal Reserve Bank of Minneapolis Quarterly Review, 25(1), 2-11.

Ayres and Nicolini (forthcoming)

Cochrane, J. (2011): "Determinacy and Identification with Taylor Rules," Journal of Political Economy, 119(3), 565-615.

Fisher, I. (1926): "A Statistical Relationship Between Unemployment and Price Changes," International Labour Review, 13(6), 785-92. Reprinted in 1973 as "I Discovered the Phillips Curve," Journal of Political Economy, 81(2): 496-502.

Fisher, J. D. M., C. T. Liu, and R. Zhou (2002): "When Can We Forecast Inflation?" Federal Reserve Bank of Chicago Economic Perspectives, Q1, 30-42.

Galí, J. (2008): Monetary Policy, Inflation, and the Business Cycle: An Introduction to the New Keynesian Framework, Princeton, NJ: Princeton University Press.

Lucas, R. E., Jr. (1972): "Expectations and the Neutrality of Money," Journal of Economic Theory, 4(2), 103-24.

Nicolini, J. P. (2013): "Monetary Policy and the Quantity Theory of Money," Mimeo. Research Department, Federal Reserve Bank of Minneapolis.

Phillips, A. W. (1958): "The Relation Between Unemployment and the Rate of Change of Money Wage Rates in the United Kingdom, 1861-1957," Economica, 25(100), 283-99.

Reifschneider, D., R. Tetlow, and J. Williams (1999): "Aggregate Disturbances, Monetary Policy, and the Macroeconomy: The FRB/US Perspective," Federal Reserve Bulletin, January. 
Samuelson, P. A., and R. M. Solow (1960): "Analytical Aspects of Anti-Inflation Policy," American Economic Review, 50(2), 177-94.

Sargent, T. J. (1999): The Conquest of American Inflation, Princeton, NJ: Princeton University Press.

Taylor, J. B. (1999): "The Robustness and Efficiency of Monetary Policy Rules as Guidelines for Interest Rate Setting by the European Central Bank," Journal of Monetary Economics, 43, 655-79.

Woodford, M. (2003): Interest and Prices: Foundations of a Theory of Monetary Policy, Princeton, NJ: Princeton University Press. 